\title{
«Une histoire de la manière dont les choses font problème »
}

Entretien de Michel Foucault avec André Berten (7 mai 1981)

Michel Foucault

\section{(2) OpenEdition}

\section{Journals}

\section{Édition électronique}

URL : http://journals.openedition.org/conflits/18897

DOI : $10.4000 /$ conflits. 18897

ISSN : $1777-5345$

Éditeur :

CCLS - Centre d'études sur les conflits lilberté et sécurité, L'Harmattan

Édition imprimée

Date de publication : 31 décembre 2014

Pagination : 99-109

ISBN : 978-2-343-05760-6

ISSN : 1157-996X

\section{Référence électronique}

Michel Foucault, « « Une histoire de la manière dont les choses font problème » », Cultures \& Conflits

[En ligne], 94-95-96 | été-automne-hiver 2014, mis en ligne le 20 février 2016, consulté le 30 mars 2021. URL : http://journals.openedition.org/conflits/18897; DOI : https://doi.org/10.4000/conflits. 18897 


\title{
«Une histoire de la manière dont les choses font problème»
}

\author{
Entretien de Michel Foucault avec André Berten (7 mai 1981)
}

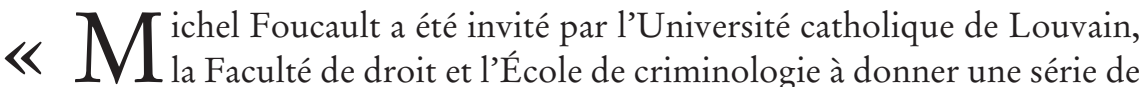
cours-conférences qu'il a intitulée “Mal faire, dire vrai” sur la fonction de l'aveu en justice. Pour vous présenter à nos auditeurs, je souhaiterais vous poser un certain nombre de questions. Bien sûr, vous êtes très connu, vous donnez cours au Collège de France, vous avez publié une série d'ouvrages depuis L'histoire de la folie, Naissance de la clinique, Les mots et les choses, L'ordre du discours, L'archéologie du savoir, Surveiller et punir-, et vous êtes en train d'écrire une Histoire de la sexualité. Ces ouvrages sont pour la plupart connus, certains plus que d'autres ; certains ont suscité des débats passionnés. Mais il me semblerait intéressant que vous puissiez nous dire comment vous avez cheminé à travers une série de problématiques, une série de questions - pourquoi vous vous êtes intéressé à l'histoire de la psychiatrie, à l'histoire de la médecine, à la prison et maintenant à l'histoire de la sexualité, pourquoi aujourd'hui vous vous intéressez à l'histoire du droit. Quel a été, au fond, votre itinéraire ? Quel a été le fil conducteur de votre réflexion, s’il est possible de répondre à une telle question?

Michel Foucault (M.F.) - C'est une question difficile que vous me posez. D'abord, parce que le fil conducteur, on ne peut guère le dégager qu'une fois qu'on a été conduit au terme dernier, c'est-à-dire au moment où on va cesser d'écrire. Et puis au fond, vous savez, je ne me considère absolument ni comme un écrivain, ni comme un prophète. Je travaille, c'est vrai, en grande partie, souvent, au gré de circonstances, de sollicitations extérieures, de conjonctures diverses, et je n'ai pas du tout l'intention de faire la loi. Et il me semble que s'il y a dans ce que je fais une certaine cohérence, elle est peut-être plus liée à une situation qui nous appartient à tous, aux uns et aux autres, dans laquelle nous sommes tous pris, plus qu'à une intuition fondamentale ou à une pensée systématique. C'est vrai, si vous voulez, il me semble, que la philosophie moderne, peut-être depuis le jour où Kant a posé la question "Was ist Aufklärung ? », c'est- à-dire : "Qu'est-ce que c'est que notre actualité ? 
Qu'est-ce qui se passe autour de nous ? Qu'est-ce que c'est que notre présent ? ", il me semble que la philosophie a acquis là une dimension, ou que s'est ouverte pour elle une certaine tâche qu'elle avait ignorée ou qui n'existait pas pour elle auparavant, qui est de dire qui nous sommes, de dire qu'est-ce que c'est que notre présent, qu'est-ce que c'est que ça, aujourd'hui. C'est évidemment une question qui n'aurait pas eu de sens pour Descartes. C'est une question qui commence à avoir du sens pour Kant quand il se demande ce que c'est que l'Aufklärung. C'est une question qui est, en un sens, la question de Hegel : «Qu'est-ce que c'est que le maintenant ? . Et c'est la question, aussi, de Nietzsche. Je pense que la philosophie, parmi les différentes fonctions qu'elle peut et qu'elle doit avoir, a aussi celle-là, de s'interroger sur ce que nous sommes dans notre présent et dans notre actualité. Je dirais que c'est en un sens autour de cela que je pose la question - et, dans cette mesure, je suis nietzschéen ou hégélien ou kantien par ce côté-là.

Alors, comment est-ce que j'en suis venu à poser ce genre-là de questions ? Pour faire deux mots d'histoire de notre vie intellectuelle aux uns et aux autres dans l'Europe occidentale après la guerre, on pourrait dire ceci. D’une part, on disposait, vers les années 1950, d'une perspective, d'un mode d'analyse très profondément inspiré par la phénoménologie qui était en un sens, à ce moment-là, une philosophie, je dirais, dominante. Dominante, je ne mets aucune péjoration dans ce mot-là : on ne peut pas dire qu'il y avait une dictature ou un despotisme de ce mode-là de pensée - mais, dans l'Europe occidentale, en particulier en France, la phénoménologie était un peu un style général d'analyse. C'était un style d'analyse qui revendiquait comme l'une de ses tâches fondamentales l'analyse du concret. Et il est très certain que de ce point de vue-là, on pouvait rester un petit peu insatisfait, dans la mesure où le concret auquel se référait la phénoménologie était - comment dire ? - un petit peu académique et universitaire. Vous aviez des objets privilégiés de la description phénoménologique qui étaient des expériences vécues : la perception d'un arbre à travers la fenêtre du bureau... Enfin, je suis un peu sévère, mais le champ d'objets que la phénoménologie parcourait était un peu prédéterminé par une tradition philosophique et universitaire qu'il valait peut-être la peine d'ouvrir un petit peu. Deuxièmement, autre forme de pensée dominante, importante, c'était évidemment le marxisme - qui, lui, se référait à tout un domaine d'analyse historique sur lequel, en un sens, il faisait l'impasse. Autant la lecture des textes de Marx, l'analyse des concepts de Marx, était une tâche importante, autant les contenus historiques, le savoir historique auquel ces concepts devaient se rapporter, pour lesquels ils devaient être opératoires, ces domaines historiques étaient un petit peu laissés pour compte. En tout cas, une histoire marxiste concrète n'était pas, en France du moins, très développée. Puis, il y avait un troisième courant qui était, en France, très spécialement développé et qui était l'histoire des sciences, avec des gens comme Bachelard, Canguilhem, Cavaillès avant-guerre. Le problème était de savoir s'il y a une historicité de la raison et si l'on peut faire l'histoire de la vérité. 
Si vous voulez, je dirais que je me suis situé un peu au croisement de ces différents courants et de ces différents problèmes. En me posant, par rapport à la phénoménologie, la question suivante : plutôt que de faire la description un peu intériorisée de l'expérience vécue, est-ce qu'il ne faut pas, est-ce qu'on ne peut pas faire l'analyse d'un certain nombre d'expériences collectives et sociales ? La conscience de celui qui est fou est importante à décrire, comme l'avaient montré les travaux de Binswanger et de Kuhn. Mais après tout, est-ce qu'il n'y a pas une structuration culturelle et sociale de l'expérience de la folie, et est-ce que cela ne doit pas être analysé ? Ce qui m'amenait à recouper un problème historique : (... ${ }^{1}$ si l'on veut décrire l'articulation sociale et collective d'une expérience comme celle de la folie, quel est le champ social, quel est l'ensemble des institutions et pratiques qu'il faut historiquement analyser et pour lesquelles les analyses marxistes sont un petit peu comme des habits de confection mal ajustés à ces développements ? Et troisièmement, à travers ça à travers l'analyse d'expériences historiques, collectives, sociales, liées à des contextes historiques précis -, comment est-ce qu'on peut faire l'histoire d'un savoir, l'histoire de l'émergence d'une connaissance, et comment des objets nouveaux peuvent arriver dans le domaine de la connaissance, peuvent se présenter comme objets à connaître ?

Alors, si vous voulez, concrètement, cela donne ceci. Est-ce qu'il y a ou non une expérience de la folie caractéristique d'une société ou d'un type de société comme les nôtres ? Comment cette expérience de la folie a pu se constituer, comment est-ce qu'elle a pu émerger ? Et, à travers cette expérience de la folie, comment est-ce que la folie a pu se constituer comme objet de savoir pour une médecine qui se présentait comme médecine mentale ? Ce qui donne, en gros : à travers quelles transformations historiques, quelles modifications institutionnelles, s'est constituée une expérience de la folie dans laquelle il y a à la fois le pôle subjectif de l'expérience de la folie et ce pôle objectif de la maladie mentale?

Voilà un petit peu, sinon l'itinéraire, du moins le point de départ. Et pour en revenir un petit peu à la question que vous me posiez ( Pourquoi avoir pris ces objets-là ?»), je dirais qu'il me semblait... Et alors, c'était peut-être là le quatrième courant, le quatrième point de référence de mon cheminement ou de mon piétinement - ça a été, si vous voulez, des textes plus littéraires, moins intégrés dans la tradition philosophique (je pense à des écrivains comme Blanchot, comme Artaud, comme Bataille, qui ont été pour des gens de ma génération, je crois, très importants) -, qui était, au fond, la question des expériences limites. Ces formes d'expérience qui au lieu d'être considérées comme centrales et d'être valorisées positivement dans une société, sont considérées comme les expériences limites, les expériences frontières à partir de quoi est remis en question cela même qui est considéré d'ordinaire comme acceptable.

1. La phrase enregistrée commence par « est-ce que l'on peut». 
Alors, en un sens, faire de l'histoire de la folie une interrogation sur notre système de raison...

André Berten (A.B.) - Comme une expérience... La folie comme une expérience limite...

M.F. - C’est ça. Par exemple, quel est le rapport entre la pensée médicale le savoir de la maladie et de la vie - qu'est-ce que c'est par rapport à l'expérience de la mort ? Et comment est-ce que le problème de la mort a été intégré dans ce savoir, ou comment ce savoir a été indexé à ce moment, ce point absolu de la mort ? Même chose pour le crime par rapport à la loi : au lieu d'interroger la loi elle-même, et qu'est-ce qui peut fonder la loi, prendre le crime comme le point de rupture par rapport au système, et prendre ce point de vuelà pour interroger : "Qu'est-ce que c'est donc que la loi ? » Prendre la prison comme ce qui doit nous éclairer sur ce que c'est que le système pénal plutôt que de prendre le système pénal, de l'interroger de l'intérieur, de savoir comment il s'est fondé, comment il se fonde et se justifie, pour en déduire ensuite ce qu'a été la prison.

A.B. - Vous avez présenté la philosophie dans son actualité - au fond, depuis Kant - comme posant une question qui est une question, je pense, qui nous concerne tous, et qui permet au fond à l'bomme de s'interroger sur sa situation dans l'bistoire, dans le monde, dans la société. Il me semble qu'à travers tout ce que vous avez écrit depuis l'Histoire de la folie jusqu'à l'Histoire de la sexualité, il y a une perception de cette réalité... Ou, du moins, il y a la perception d'un élément de cette réalité qui semble vous intéresser particulièrement et qui concerne tout ce qu'on pourrait appeler les techniques d'enfermement, de surveillance, de contrôle - bref, la manière dont l'individu dans notre société a été progressivement contrôlé. Est-ce que vous pensez qu'effectivement, il s'agit là d'un élément qui, déjà depuis un certain temps, depuis peutêtre l'époque classique dans notre histoire, est très déterminant pour comprendre notre modernité?

M.F. - Oui, c'est vrai. Si vous voulez, ce n'est pas un problème que je m'étais posé au départ. C'est petit à petit, en étudiant un certain nombre de choses - comme justement la psychiatrie, la médecine, le système pénal - que tous ces mécanismes, d'enfermement, d'exclusion, de surveillance, de contrôle individuel, m'ont paru très intéressants et très importants. Je dirais que j'ai peut-être posé ces questions-là d'une façon un peu sauvage à un moment donné, au moment où je me suis aperçu qu'elles étaient importantes. Je crois qu'il faut bien délimiter de quoi il s'agit et quel est le genre de problème que l'on peut poser à propos de tout cela. Il me semble que dans la plupart des analyses, soit des analyses de type proprement philosophique, soit dans des analyses plus politiques sinon des analyses marxistes, la question du pouvoir avait été relativement marginalisée... 


\section{A.B - Simplifiée...}

M.F. - Ou en tout cas, simplifiée. Ou bien il s'agissait de savoir quels étaient les fondements juridiques pouvant légitimer un pouvoir politique, ou bien il s'agissait de définir le pouvoir dans une fonction de simple conservation ou reconduction de rapports de production. Alors, il s'agissait de la question philosophique du fondement ou de l'analyse historique de la superstructure. Ceci me paraissait insuffisant, m'a paru insuffisant pour un certain nombre de raisons. D'abord parce que, je crois - et un certain nombre de choses dans des domaines un peu concrets que j'ai essayé d'analyser le montrent -, les rapports de pouvoir sont beaucoup plus profondément implantés qu'au simple niveau des superstructures. Deuxièmement, la question du fondement du pouvoir est importante, mais - je m'excuse - le pouvoir ne fonctionne pas à partir de son fondement. Il y a des pouvoirs non fondés qui fonctionnent très bien et des pouvoirs qui ont cherché à se fonder, qui se sont effectivement fondés et qui, finalement, n'ont pas fonctionné. Donc, si vous voulez, mon problème a été de me dire : "Mais ne peut-on pas étudier la manière dont, effectivement, fonctionne le pouvoir ? »

Quand je dis « le pouvoir », il ne s'agit absolument pas de repérer une instance ou une espèce de puissance qui serait là, occulte ou visible - peu importe -, et qui diffuserait son rayonnement nocif à travers le corps social ou qui étendrait d'une façon fatale son réseau. Il ne s'agit pas pour le pouvoir, ou pour quelque chose qui serait « le pouvoir ", de jeter un grand filet de plus en plus serré qui étranglerait et la société, et les individus. Ce n'est absolument pas de cela qu'il s'agit. Le pouvoir, c'est des relations. Le pouvoir, ce n'est pas une chose. C'est une relation entre deux individus, et une relation qui est telle, que l'un peut conduire la conduite d'un autre ou déterminer la conduite d'un autre - la déterminer volontairement en fonction d'un certain nombre d'objectifs qui sont les siens. Autrement dit, quand on regarde ce qu'est le pouvoir, c'est l'exercice de quelque chose qu'on peut appeler le gouvernement, au sens très large. on peut gouverner une société, on peut gouverner un groupe, on peut gouverner une communauté, on peut gouverner une famille, on peut gouverner quelqu'un. Et quand je dis gouverner quelqu'un, c'est simplement au sens où l'on peut déterminer sa conduite en fonction de stratégies en utilisant un certain nombre de tactiques. Donc, si vous voulez, c'est la gouvernementalité au sens large, entendue comme ensemble des relations de pouvoir et techniques qui permettent à ces relations de pouvoir de s'exercer, c'est cela que j'ai essayé d'étudier. Comment est-ce qu'on a gouverné les fous, comment est-ce que le problème du gouvernement des malades - et encore une fois, je mets le mot « gouvernement » entre guillemets, en lui donnant à la fois un sens large et riche... Comment est-ce qu'on a gouverné les malades, qu'est-ce qu'on en a fait, quel statut on leur a donné, où est-ce qu'on les a placés, dans quel système de traitement, de surveillance aussi, de bienveillance, de philanthropie, dans quel champ économique les soins à apporter aux malades... 
C’est tout cela, je crois, qu'il faut essayer de voir.

Alors, c'est certain que cette gouvernementalité n'a pas cessé, d'un certain point de vue, de devenir plus stricte au cours des âges. Le pouvoir, dans un système politique comme ceux qu'on a connus au Moyen-Âge, le pouvoir entendu au sens de gouvernement des uns par les autres, était finalement assez lâche : le problème était de faire le prélèvement fiscal qui était nécessaire, qui était utile ou qu'on voulait... Ce que les gens faisaient dans leur conduite quotidienne n'était pas très important pour l'exercice du pouvoir politique (c'était important, sans doute, dans le pastorat ecclésiastique). Pour le pouvoir politique, il est venu un moment où ça a été très important - et où par exemple maintenant, pour prendre un exemple très simple, le type de consommation des gens devient quelque chose qui est important économiquement, qui est important aussi politiquement. Et c'est vrai que le nombre d'objets qui deviennent objets d'une gouvernementalité réfléchie à l'intérieur de cadres politiques - même libéraux -, ce nombre d'objets a considérablement augmenté. Mais je ne pense pas non plus qu'il faille considérer que cette gouvernementalité prenne forcément la forme de l'enfermement, de la surveillance et du contrôle. Par toute une série d'interventions, subtiles souvent, on arrive en effet à conduire la conduite des gens ou à se conduire de telle manière que la conduite des autres ne puisse pas avoir les effets nocifs qu'on redoute. C'est tout ce champ de la gouvernementalité que j'ai voulu étudier.

A.B - Pour étudier cet objet ou ces différents objets, vous avez utilisé une méthode historique. Mais ce qui apparaît à tous aujourd'bui-et d'ailleurs, ce qui fait en grande partie la nouveauté de vos analyses, non pas tant du point de vue du contenu, mais du point de vue de la méthode -, c'est que vous avez opéré une sorte de déplacement dans la méthode historique. Ce n'est plus une bistoire de la science, ce n'est plus une épistémologie, ce n'est plus une histoire des idéologies, ce n'est même pas une histoire des institutions. On a l'impression que c'est tout cela en même temps, mais que pour penser par exemple le travail de la psychiatrie, on ce que font aujourd'bui les criminologues (puisque ce sont les criminologues qui vous ont appelé ici), ou pour penser des institutions

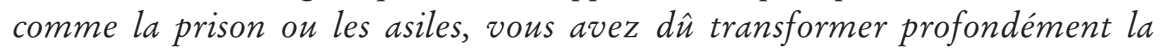
manière dont on concevait l'histoire. Est-ce que, par exemple, l'opposition entre savoir et science qui apparaît dans votre œuvre, principalement dans un certain nombre d'écrits plus méthodologiques, est-ce qu'elle vous semble très importante du point de vue de ce genre d'histoire que vous nous proposez?

M.F. - Je crois, en effet, que le type d'histoire que je fais porte un certain nombre de marques ou de handicaps, comme vous voudrez. D'abord, la chose que je voudrais dire, c'est que, encore une fois, la question dont je pars est "Qu'est-ce que nous sommes, et qu'est-ce que nous sommes aujourd'bui? Qu'est-ce que c'est que cet instant qui est le nôtre ? " Donc, si vous voulez, 
c'est une histoire qui part bien de cette actualité. Deuxième chose, c'est qu'en essayant de poser des problèmes concrets, je pars forcément, et ce qui me paraît intéressant, c'est de prendre, de choisir comme domaines des points qui semblent particulièrement fragiles ou sensibles dans l'actualité. C'est-à-dire que je ne concevrais guère une histoire, si vous voulez, qui soit proprement spéculative et dont le champ ne soit pas déterminé par quelque chose qui se passe actuellement. Tout l'intérêt, c'est non pas bien sûr de suivre ce qui se passe et de suivre, comme on dit, la mode - par exemple, une fois qu'on a écrit dix bouquins, très bons d'ailleurs, sur la mort, on ne va pas en écrire un onzième sous prétexte que c'est la question actuelle. Il s'agit de... Le jeu, c'est d'essayer de détecter, parmi les choses dont on n'a pas encore parlé, quelles sont celles qui actuellement présentent, montrent, donnent quelques indices plus ou moins diffus de fragilité dans notre système de pensée, dans notre mode de réflexion, dans notre pratique.

Il y avait, si vous voulez, vers les années 1955 quand je travaillais dans des hôpitaux psychiatriques, il y avait une espèce de crise latente, quelque chose dont on sentait bien que ça s'écaille, et on n'en avait pas encore beaucoup parlé. Mais, pourtant, ça se vivait assez intensément - la meilleure preuve que ça se vivait, c'est qu'à côté, en Angleterre, sans qu'on ait eu jamais aucun rapport les uns avec les autres, des gens comme Laing et Cooper, eux aussi, se battaient avec le même problème. Donc, c'est une histoire qui se réfère toujours à une actualité. Même chose pour le problème de la médecine. C'est vrai que la question du pouvoir médical - ou en tout cas du champ institutionnel à l'intérieur duquel le savoir médical fonctionne - était une question qui commençait à se poser, qui était même assez largement posée vers les années 1960, et qui n'est entrée dans le domaine public qu'après 1968. C'est donc une histoire de l'actualité en train de se dessiner.

A.B. - Oni, mais par rapport à cette actualité, au fond, la manière dont vous élaborez l'histoire me semble originale. Elle me semble... an fond, elle est réglée par l'objet même que vous analysez. C'est en raison de ces problèmes que vous percevez comme étant fragiles ou comme étant des problèmes-clef dans notre société que vous êtes amené à en refaire l'bistoire pour les éclairer, mais à en refaire l'histoire d'une manière spécifique...

M.F. - Alors, au niveau des objectifs que je pose en faisant cette histoire, souvent les gens ont lu ce que j'ai fait comme des sortes d'analyses à la fois compliquées, un peu obsessives, et qui mènent à ce résultat que finalement mon Dieu, comme nous sommes emprisonnés dans le système qui est le nôtre, combien sont nombreux les lacets qui nous enserrent, et combien il est difficile de dénouer les nœuds que l'histoire a noués autour de nous! Alors qu'en réalité, je fais tout le contraire! Car, lorsque j'ai essayé d'étudier quelque chose comme la folie ou la prison... Prenons, si vous voulez, l'exemple de la 
prison. Quand on discutait, il y a encore quelques années - disons, au début des années 1970 - avec les gens sur la réforme du système pénal, une chose me paraissait très frappante. C'est que, par exemple, bien sûr on posait la question théorique du droit de punir. Bien sûr, d'un autre côté, on posait le problème de savoir comment on peut aménager le régime pénitentiaire. Mais cette espèce d'évidence, si vous voulez, que la privation de liberté est au fond la forme la plus simple, la plus logique, la plus raisonnable, la plus équitable de punir quelqu'un parce qu'il avait commis une infraction, cela n'était pas tellement interrogé. Or, ce que j'ai voulu faire, c'est de montrer combien finalement cette adéquation, pour nous si claire et simple, de la peine avec la privation de liberté était en réalité quelque chose de récent. C'est une invention, une invention technique, dont les origines bien sûr sont lointaines, mais qui a vraiment été intégrée à l'intérieur du système pénal et qui a fait partie de la rationalité pénale à partir de la fin du XVIII e siècle. Et j'ai essayé d'interroger les raisons pour lesquelles la prison était ainsi devenue une sorte d'évidence dans notre système pénal.

Il s'agit donc de rendre les choses plus fragiles par cette analyse historique - ou plutôt, de montrer à la fois pourquoi et comment les choses ont pu se constituer ainsi, mais montrer en même temps qu'elles se sont constituées à travers une histoire précise. Il faut donc montrer et la logique des choses - ou, si vous voulez, la logique des stratégies à l'intérieur desquelles les choses se sont produites -, et montrer que ce ne sont pourtant que des stratégies et que, du coup, en changeant un certain nombre de choses, en changeant de stratégie, en prenant les choses autrement, du coup, ce qui nous paraissait évident ne l'est pas. Notre rapport à la folie, c'est un rapport qui est historiquement constitué. Et du moment qu'il est historiquement constitué, il peut être politiquement détruit - je dis «politiquement » en donnant au mot «politique » un sens très large. En tout cas, il y a des possibilités d'action, puisque c'est à travers un certain nombre d'actions, de réactions, à travers un certain nombre de luttes, de conflits pour répondre à un certain nombre de problèmes qu'on a choisi ces solutions-là. J'ai voulu réintégrer beaucoup des évidences de notre pratique dans l'historicité même de ces pratiques. Et du coup, les déchoir de leur statut d'évidence pour leur redonner la mobilité qu'elles ont eue et qu'elles doivent toujours avoir dans le champ de nos pratiques.

A.B. - Dans vos conférences actuelles, vous utilisez le terme "véridiction", qui se réfère à un "dire la vérité » et qui touche à un problème de vérité. Dans la méthode et dans ce que vous venez de dire concernant à la fois votre intérêt pour l'actualité et la manière dont vous envisagez l'histoire et la constitution même de cette actualité, en quelque sorte vous remettez en question ce qu'on pourrait penser être les fondements de telle ou telle pratique. Vous nous avez dit à propos du pouvoir, [que] le pouvoir ne fonctionne pas à partir de son fondement, mais [qu']il $y$ a effectivement toujours des justifications ou des 
réflexions philosophiques qui visent à fonder le pouvoir. Votre méthode historique, qui est une méthode qui fait une sorte d'archéologie ou de généalogiepeut-être selon les objets ou selon le développement même de votre pensée-, vise à montrer que finalement, il n'y a pas de fondement des pratiques de pouvoir. Est-ce que vous seriez d'accord pour dire, d'un point de vue philosophique et dans l'ensemble de votre développement, que ce que vous visez, c'est aussi à déconstruire toute entreprise qui viserait à donner un fondement au powvoir?

M.F. - Mais je crois que l'activité de donner un fondement au pouvoir, l'activité qui consiste à s'interroger sur ce qui fonde le pouvoir que j'exerce ou ce qui peut fonder le pouvoir qui s'exerce sur moi, je crois que cette question est importante. Elle est essentielle. Je dirais que c'est la question qui est fondamentale. Mais le fondement que l'on donne en réponse à cette question fait partie d'un champ historique à l'intérieur duquel elle a une place tout à fait relative. C'est-à-dire qu'on ne trouve pas de fondement, mais il est très important que, dans une culture comme la nôtre - la question est de savoir si on peut le trouver ailleurs ou pas, dans d'autres cultures, cela, je n'en sais rien... Mais il est très important que pour nous, depuis non seulement des siècles, mais des millénaires, un certain nombre de choses, comme en particulier l'exercice du pouvoir politique, s'interroge lui-même ou se voie interrogé par des gens qui posent la question : "Mais qu'est-ce qui vous fonde ? Qu'est-ce qui vous légitime ? ». Il y a là un travail critique...

A.B. - Ce que vous trouvez important, c'est justement le travail critique de cette question qui sans cesse revient, et qui interroge la vérité...

M.F. - C'est ça : qui sans cesse revient. Il y a deux millénaires qu'on s'interroge sur le fondement du pouvoir politique - quand je dis deux millénaires, c'est deux millénaires et demi ; on s'interroge sur le pouvoir politique, et c'est cette interrogation qui est fondamentale.

A.B. - Et au fond, le type d'bistoire que vous avez faite, c'est bien une analyse, vous avez dit des stratégies, mais aussi une analyse de la manière dont un certain nombre de pratiques ont recherché leur propre fondement.

M.F. - Absolument. Absolument. Je dirais que c'est l'histoire des - je vais employer encore un mot barbare, mais les mots ne sont barbares que lorsqu'ils ne disent pas bien clairement ce qu'ils veulent dire, si bien que beaucoup de mots familiers sont barbares, parce qu'ils disent beaucoup de choses à la fois ou qu'ils ne disent rien, et qu'en revanche, certains mots techniques bizarrement construits ne sont pas barbares s'ils disent à peu près clairement ce qu'ils veulent dire... Je dirais que je fais l'histoire des problématisations, c'est-à-dire l'histoire de la manière dont les choses font problème. Comment 
et pourquoi et sur quel mode particulier la folie a-t-elle fait problème dans le monde moderne ? Et pourquoi est-ce que c'est devenu un problème important ? Problème tellement important qu'un certain nombre de choses, par exemple comme la psychanalyse - qui, Dieu sait, a diffusé à travers toute notre culture - est tout de même partie d'un problème qui était un problème absolument intérieur au rapport que l'on pouvait avoir avec la folie. C'est l'histoire de ces problèmes. sur quel mode nouveau la maladie - qui, évidemment, a toujours fait problème, mais il y a eu un mode nouveau de problématisation de la maladie, me semble-t-il, à partir du XVIII' et du XIXe siècle...

Alors ce n'est, en effet, ni l'histoire des théories, ni l'histoire des idéologies, ni même l'histoire des mentalités qui m'intéresse. C'est l'histoire des problèmes, c'est si vous voulez la généalogie des problèmes qui m’intéresse : pourquoi un problème, et pourquoi tel type de problème, pourquoi tel mode de problématisation apparaît à un certain moment à propos d'un domaine. Par exemple, à propos de la sexualité, j'ai mis très longtemps à commencer à apercevoir comment on pouvait répondre à cela : quel a été le nouveau problème ? Voyez-vous, à propos de la sexualité, ce n'est pas tellement de savoir et de répéter indéfiniment la question : «Voyons, est-ce que c'est le christianisme, ou est-ce que c'est la bourgeoisie, ou est-ce que c'est l'industrialisation, qui a amené la répression de la sexualité ? » La répression de la sexualité n'est intéressante que dans la mesure où, d'une part, elle fait souffrir un certain nombre de gens, encore aujourd'hui ; elle est intéressante d'autre part parce qu'elle a toujours pris des formes diverses, mais ayant toujours existé. Ce qui me paraît quelque chose d'important à faire apparaître, c'est comment et pourquoi ce rapport à la sexualité ou ce rapport à nos comportements sexuels ont fait problème, et sous quelles formes ils ont fait problème. Car ils ont toujours fait problème, mais il est certain qu'ils n'ont pas fait problème de la même façon chez les Grecs du IV siècle avant Jésus- Christ, chez les chrétiens du III ${ }^{\mathrm{e}}-\mathrm{IV}^{\mathrm{e}}$ siècle, au XVIe-XVII ${ }^{\mathrm{e}}$ siècle, maintenant... C'est cette histoire des problématisations. Alors, comment, dans les pratiques humaines, il y a un moment où en quelque sorte les évidences se brouillent, les lumières s'éteignent, le soir se fait, et où les gens commencent à s'apercevoir qu'ils agissent à l'aveugle et que, par conséquent, il faut une nouvelle lumière. Il faut une nouvelle lumière, il faut un nouvel éclairage, et il faut de nouvelles règles de comportement. Et alors, voilà qu'un nouvel objet apparaît, un objet qui apparaît comme problème. Voilà.

A.B - Je voudrais vous poser une dernière question. Vous avez été invité par la Faculté de droit et vous semblez vous intéresser particulièrement maintenant au droit et au phénomène juridique. Est-ce que vous pourriez expliquer brièvement d'où vient cet intérêt pour le droit, et ce que vous espérez en retirer? 
M.F. - Écoutez, je crois que je me suis toujours un peu intéressé au droit. Comme un laïc : je ne suis pas un spécialiste du droit, je ne suis pas un juriste. Mais aussi bien à propos de la folie qu'à propos du crime ou de la prison, $j$ 'ai rencontré le problème du droit, le problème de la loi. Et la question que j'ai toujours posée, c'était de savoir comment les technologies de gouvernement, comment ces rapports de pouvoir entendus au sens où nous l'avons dit tout à l'heure, comment tout cela pouvait prendre forme à l'intérieur d'une société qui prétend fonctionner au droit et qui, pour une part au moins, fonctionne au droit. Alors ce sont les liens, les rapports de cause à conséquence, les conflits aussi, les oppositions, les irréductibilités entre ce fonctionnement du droit et cette technologie du pouvoir, c'est ça que je voudrais étudier. Et il me semble qu'interroger les institutions juridiques, interroger le discours et la pratique du droit à partir de ces technologies de pouvoir peut présenter un certain intérêt - non pas du tout au sens que cela bouleverserait entièrement et l'histoire et la théorie du droit, mais il me semble que cela peut jeter une certaine lumière sur certains aspects assez importants de la pratique et de la théorie judiciaires. Ainsi, interroger le système pénal moderne à partir de la pratique punitive, de la pratique corrective, à partir de toutes ces technologies par lesquelles on a voulu modeler, modifier, et caetera, l'individu criminel, il me semble que ça permet sans doute de faire apparaître un certain nombre de choses. Donc, si vous voulez, je croise sans cesse le droit sans le prendre comme objet particulier. Et si Dieu me prête vie, après la folie, la maladie, le crime, la sexualité, la dernière chose que je voudrais étudier, eh bien, ce serait le problème de la guerre et de l'institution de la guerre dans ce qu'on pourrait appeler la dimension militaire de la société. Et là encore, j'aurai à croiser le problème du droit, aussi bien sous la forme du droit des gens, du droit international et caetera, qu'aussi le problème de la justice militaire et de ce qui fait qu'une nation peut demander à quelqu'un de mourir pour elle.

A.B. - Eh bien, nous espérons tous que Dieu vous prêtera vie pour pouvoir continuer à lire vos histoires, ces histoires multiples qui nous ont tant enrichis. Je vous remercie.

M.F. - Je ne lui souhaite pas. 